\title{
Myocardial injuries among patients with COVID-19: a systematic review
}

\author{
Alaa Hasan Alali', Mustafa Samir Smaisem², Ahmed Mohammed Alsheikh², \\ Aljohara Abdullah Alshareef², Fatema Samir Smaisem², Batool Wael Alnahar², \\ Amal Khalil Hassouneh ${ }^{3}$, Jaffar A. Al-Tawfiq ${ }^{4,5,6}$, Ziad A. Memish ${ }^{7,8,9}$ \\ ${ }^{1}$ Internal Medicine and Infectious Diseases, King Saud Medical City, Riyadh, Saudi Arabia; \\ ${ }^{2}$ Almaarefa University, Riyadh, Saudi Arabia; \\ IInfectious diseases Clinical Pharmacist, King Saud Medical City, Riyadh, Saudi Arabia; \\ ${ }^{4}$ Specialty Internal Medicine and Quality Department, Johns Hopkins Aramco Healthcare, Dhahran, Saudi Arabia; \\ Infectious Disease Division, Indiana University School of Medicine, Indiana, USA; \\ Infectious Disease Division, Johns Hopkins University School of Medicine, Baltimore, MD, USA; \\ ${ }^{7}$ Research and Innovation Center, King Saud Medical City; \\ ${ }^{8}$ Faculty of Medicine, Alfaisal University, Riyadh, Saudi Arabia; \\ ${ }^{9}$ Hubert Department of Global Health, Rollins School of Public Health, Emory University, Atlanta, GA, USA
}

Article received 1 May, 2021; accepted 15 July, 2021

\section{SUMMARY}

This is a systematic review of the literature specifically aimed to explore myocardial injury in coronavirus disease-19 (COVID-19) patients who were hospitalized with severe complicated infections.

The medical literature was examined through the large medical databases, including Medline, Ovid, PubMed, and Embase, over the last year between January 2020 and May 2021. The search terms used were a combination of "myocardial injury" AND "COVID-19" AND "Hospitalization". Then we applied a step to filter the results to select original research articles only evaluating the myocardial injuries in severe COVID-19 hospitalized patients. Selected trials mentioned the type of myocardial injury detected with the infection. A total of 245 articles were extracted. Considering the exclusion of ineligible articles, 42 articles appeared.

A total of 42 articles were eligible and were included in the review. These studies included a total of 4326 COVID-19 patients. The 30-day mortality was found to be associated with increased cardiac troponin and myocardial infarction could be a systemic reaction rather than the direct action of COVID-19. Patients with myocardial injury were significantly older and with co-morbid conditions. Studies also found a correlation of higher concentrations of cardiac enzymes with disease severity and increased in-hospital mortality.

Myocardial injury was a significant predictor for severe COVID-19 infection and in-hospital mortality. Cardiac enzymes should be monitored in hospitalized patients with severe COVID-19 infections.

Keywords: cardiac, injuries, hospitalized, severe, COVID-19.

\section{INTRODUCTION}

A $t$ the end of 2019, a cluster of pneumonia of unknown etiology was reported from $\mathrm{Wu}-$ han, China. The causative virus was identified as a coronavirus and named severe acute respirato-

Corresponding author

Ziad A. Memish

E-mail: zmemish@yahoo.com ry syndrome coronavirus-2 (SARS-CoV-2) and the disease as coronavirus disease 2019 (COVID-2019) $[1,2]$. Coronaviruses are a large family of RNA viruses known to cause disease in human since the 1960s in the form of mild upper respiratory tract infections. Over the last decades, three strains emerged with significant global public health impact: SARS-CoV-1 emerged in China in 2003, Middle East respiratory coronavirus (MERS-CoV) emerged in Saudi Arabia in September 2012 and the new SARS-CoV-2 [3-9]. 
All coronaviruses are linked in their origin to bats and use animal reservoirs in their spread to humans. For SARS-CoV-1 civet cats were suspected as the animal reservoir while camels were confirmed as the animal reservoir for MERS-CoV [10]. The homology of SARS-CoV-2 to SARS-CoV in bats known as Rhinolophus affini is $96.2 \%$ indicating bats as the origin of SARS-CoV-2 [11].

SARS-CoV-2 has the ability for rapid transmission among humans and since its emergence it has caused 216,867,420 confirmed cases globally including 4,507,837 deaths as of Aug 31, 2021 as reported by the World Health Organization [12]. Like SARS-CoV-1 and MERS-CoV, COVID-19 cases affect more severely elderly patients, those immunocompromised, or with comorbidities such as diabetes mellitus, chronic kidney disease, chronic heart disease, hypertension, obesity, and ethnic minorities [13, 14]. Infections among younger age are very mild, while complications and mortality are associated with the advanced age and presence of comorbidities [15].

In patients with COVID-19, the average incubation period was 6.4 days and respiratory symptoms were the most common presenting symptoms and patients may have associated coagulopathy $[16,17]$. These clinical presentations may be related to the viral dynamics and the presence of the virus in the different tissues [18]. Additionally, severe cases may occur due to hyperinflammatory response with resultant multiple organ failure $[19,20]$. However, different studies have used different definitions for cytokine storm [21].

Although the respiratory tract is the main organ affected by COVID-19, myocardial injury has been reported specially with severe COVID-19 disease [12, 22-26]. In addition, COVID-19 tends to be more severe in the elderly who also have cardiovascular risk factors and chronic cardiovascular conditions [27]. Up to $60 \%$ of severe COVID-19 patients have concomitant cardiac injury manifested by raised troponin, electrocardiographic (ECG) abnormalities or myocardial dysfunction [28]. In a meta-analysis, patients with COVID-19 infection and elevated troponin I levels had higher odds of poor outcomes with pooled OR of 7.92 (95\% CI: 3.70-16.97; p<0.00001) [29].

Several hypotheses have been put forward to explain myocardial injury associated with COVID-19 disease including direct viral injury, myocardial injury secondary to cytokine storm, acute atherothrombosis, increase myocardial demand, hypotension, or systemic hypoxemia [30]. Therefore, we intend to review the English literature in order to explore the extent of myocardial injuries (MI) in severe COVID-19 infections among hospitalized patients.

\section{METHODOLOGY}

This is a systematic review and was conducted using PRISMA (Preferred Reporting Items for Systematic Reviews and Meta-Analyses) checklist recommendations [31]. We searched the electronic databases from January 2020 to May 2021 in four large databases: Medline, Ovid, PubMed, and Embase.

\section{Search Strategy}

The key terms used were "myocardial injury" or "cardiac injury" AND "COVID-19" AND "Hospitalization". All the titles and abstracts that were produced from this search were examined thoroughly. The results obtained were then refined to choose original research articles, mainly evaluating myocardial injury associated with COVID-19 infections in severe hospitalized patients. All study designs were considered. Only trials published in the English language were further examined in the 2nd stage of the review.

\section{Eligibility criteria}

In the second stage, abstracts were examined manually to choose the appropriate studies to be included. The inclusion criteria were having data on myocardial injury. Moreover, references of selected studies were evaluated to identify any related articles. Finally, the required data sets were gathered from the final list of eligible studies and were analysed accordingly. Articles were excluded if overlapped or with incomplete data. Moreover, the articles unavailable in the complete form or containing inappropriate research design were also excluded. Full details on the search strategy are shown in Figure 1.

\section{Data review and analysis}

The $1^{\text {st }}$ step included a preliminary review; a pre-designed excel sheet was utilized for data extraction. Selected data from the eligible studies were then revised over the excel sheet. To identify any potential duplication, the studies published 


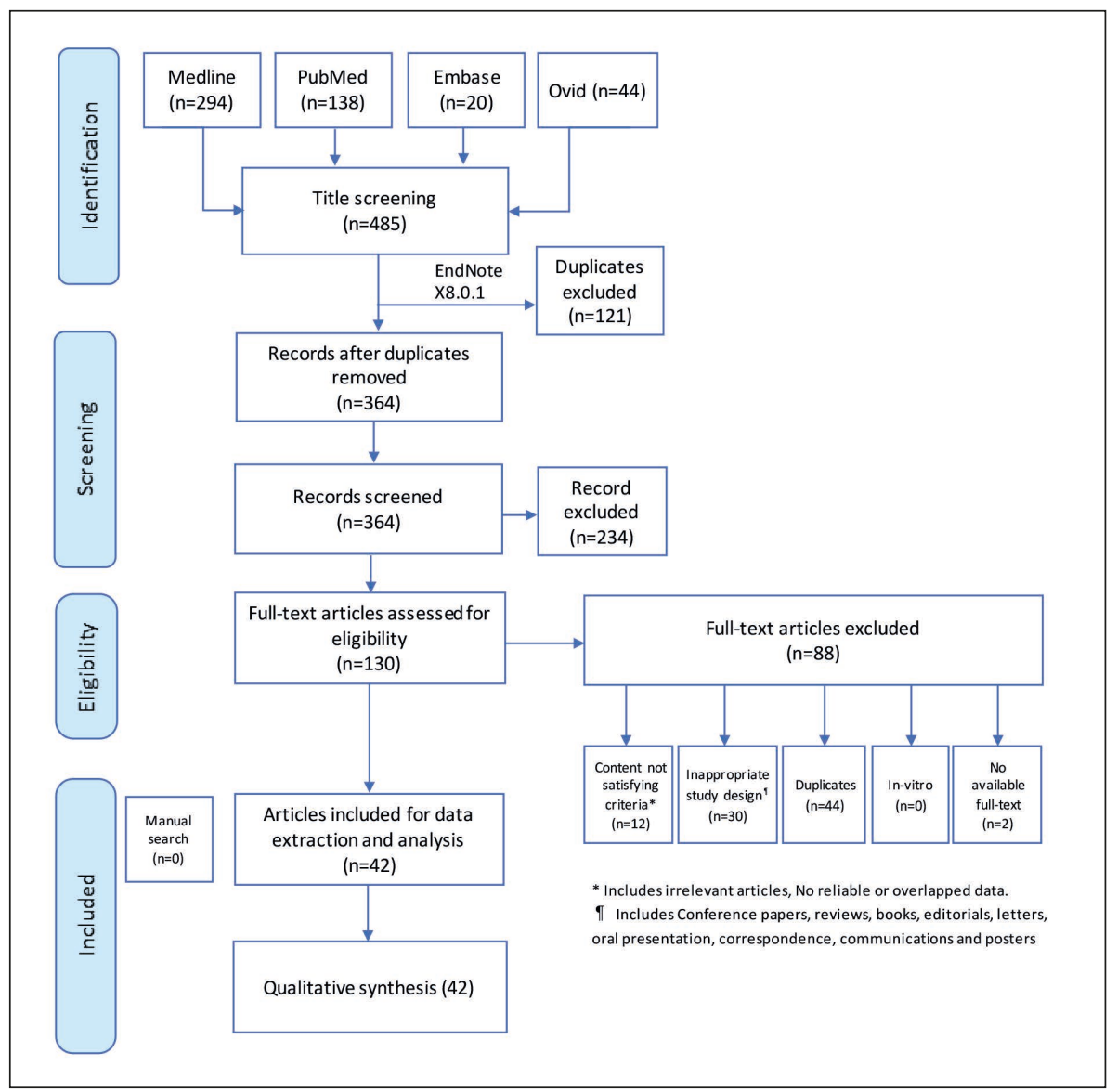

Figure 1 - Flow diagram showing the flow of the literature search and exclusion process of identified studies per the systematic review.

by the research team testing the same variables were reviewed. The team used Cochrane, i.e., a quality assessment tool, to evaluate the chosen clinical studies [32]. Data were then described statistically via frequencies (the total number of cases) along with percentages specifically for categorical variables.

\section{RESULTS}

After analyzing the abstracts and checking for the predetermined eligibility criteria, a total of 42 articles were included in the present systematic review [33-77]. Those articles were published between March 2020 and May 2021, covering a total of 4326 COVID-19 patients with myocardial injury. These studies examined various aspects of cardiac or myocardial injury among hospitalized patients or patients admitted to the intensive care units. Of all the included studies, 12 (28.5\%) were prospective studies $[33,35,37,40,42,45,49,55$, $60,70,72,76]$.

The 30-day mortality was found to be associated with increased cardiac troponin I (cTnI) $(45.1 \%$ vs. $23.2 \%$; $p=0.005$ ) [33]. Another study showed that $15.8 \%$ of admitted COVID-19 had acute myocardial injury and elevated high-sensitivity troponin $\mathrm{T}$ (hs-TnT) was associated with increased COVID-19 severity and mortality [34]. One study showed that $12.5 \%$ of patients had myocardial injury with abnormalities similar to myocarditis and that the majority had normal troponin upon admission and increasing troponin levels in 37.5\% during hospitalization, particularly in deceased patients. Thus, it was concluded that myocardial injury is commonly linked to systemic reactions rather than the direct action of COVID-19 [45]. Of 18 patients, $56 \%$ had elevated ST-segment on admission, and the others had elevated ST-segment during hospitalization and the in-hospital mor- 
Table 1 - A summary of the Included Studies addressing COVID-19 and myocardial infarction from March 2020 to May 2021.

\begin{tabular}{|c|c|c|c|c|c|c|c|c|}
\hline Author(s) & Year & $\begin{array}{l}\text { Country } \\
\text { of study }\end{array}$ & Study design & $\begin{array}{l}\text { Sample } \\
\text { size }\end{array}$ & Type of cardiac outcome & $\begin{array}{c}\text { Age of participants } \\
\text { (Years) }\end{array}$ & Disease severity & $\begin{array}{l}\text { Male: } \\
\text { Female } \\
\text { ratio }\end{array}$ \\
\hline $\begin{array}{l}\text { Lorente- } \\
\text { Ros et al. } \\
{[33]}\end{array}$ & $\begin{array}{l}\text { June, } \\
2020\end{array}$ & $\begin{array}{l}\text { Madrid, } \\
\text { Spain }\end{array}$ & Prospective & 707 & Myocardial injury & $66.76 \pm 15.7$ & Hospitalized & 1.68 \\
\hline $\begin{array}{l}\text { Wei et al. } \\
\text { [34] }\end{array}$ & $\begin{array}{c}\text { April, } \\
2020\end{array}$ & $\begin{array}{l}\text { Sichuan, } \\
\text { China }\end{array}$ & Prospective & 101 & Acute myocardial injury & $\begin{array}{l}\text { Average age was } \\
49 \text { years }\end{array}$ & ICU & 1.14 \\
\hline $\begin{array}{l}\text { Deng et al. } \\
\text { [45] }\end{array}$ & $\begin{array}{l}\text { April, } \\
2020\end{array}$ & $\begin{array}{l}\text { Wuhan, } \\
\text { China }\end{array}$ & Prospective & 112 & $\begin{array}{l}\text { Myocardial Injury with } \\
\text { myocarditis }\end{array}$ & $\begin{array}{l}\text { The median age } \\
\text { was } 65.0 \text { years }\end{array}$ & Hospitalized & 1.04 \\
\hline $\begin{array}{l}\text { Bangalore } \\
\text { et al. } \\
{[56]}\end{array}$ & $\begin{array}{l}\text { April, } \\
2020\end{array}$ & $\begin{array}{l}\text { New } \\
\text { York, } \\
\text { USA }\end{array}$ & Retrospective & 18 & $\begin{array}{l}\text { Myocardial Injury with } \\
\text { ST-segment elevation }\end{array}$ & $\begin{array}{l}\text { The median age } \\
\text { of the patients } \\
\text { was } 63 \text { years }\end{array}$ & Hospitalized & 4.88 \\
\hline $\begin{array}{l}\text { Shi et al. } \\
\text { [67] }\end{array}$ & $\begin{array}{c}\text { March, } \\
2020\end{array}$ & $\begin{array}{l}\text { Wuhan, } \\
\text { China }\end{array}$ & $\begin{array}{l}\text { Cohort } \\
\text { prospective }\end{array}$ & 416 & $\begin{array}{l}\text { Myocardial injury } \\
\text { and mortality }\end{array}$ & $\begin{array}{l}\text { The median age } \\
\text { was } 64 \text { years }\end{array}$ & Hospitalized & 0.97 \\
\hline $\begin{array}{l}\text { Guo et al. } \\
\text { [73] }\end{array}$ & $\begin{array}{l}\text { March, } \\
2020\end{array}$ & $\begin{array}{l}\text { Wuhan, } \\
\text { China }\end{array}$ & Retrospective & 187 & $\begin{array}{l}\text { Cardiovascular disease } \\
\text { and myocardial injury }\end{array}$ & $\begin{array}{l}\text { The mean (SD) age } \\
\text { was } 58.50(14.66) \\
\text { years }\end{array}$ & Hospitalized & 0.95 \\
\hline $\begin{array}{l}\text { Han et al. } \\
{[74]}\end{array}$ & $\begin{array}{c}\text { March, } \\
2020\end{array}$ & $\begin{array}{l}\text { Wuhan, } \\
\text { China }\end{array}$ & Prospective & 273 & $\begin{array}{l}\text { Lab values related to } \\
\text { myocardial injury }\end{array}$ & $\begin{array}{l}\text { The mean age (SD) } \\
58.95(10.80)\end{array}$ & Hospitalized & 0.56 \\
\hline $\begin{array}{l}\text { Zhou et al. } \\
\text { [75] }\end{array}$ & $\begin{array}{l}\text { March, } \\
2020\end{array}$ & $\begin{array}{l}\text { Anhui, } \\
\text { China }\end{array}$ & Retrospective & 34 & $\begin{array}{l}\text { Myocardial injury in } \\
\text { severe and relatively } \\
\text { very serious COVID-19 }\end{array}$ & $\begin{array}{l}\text { General group } \\
\text { mean (SD) age was } \\
40.2(12.6) \\
\text { Severe group mean } \\
\text { (SD) age was } 60.6(3.6)\end{array}$ & Hospitalized & 1.29 \\
\hline $\begin{array}{l}\text { Liaqat } \\
\text { et al. } \\
\text { [72] }\end{array}$ & $\begin{array}{l}\text { Mar, } \\
2020\end{array}$ & Pakistan & Prospective & 201 & $\begin{array}{l}\text { Myocardial injury } \\
\text { associated with } \\
\text { abnormal ECG changes }\end{array}$ & $\begin{array}{l}\text { The mean age (SD) } \\
\text { was } 44.62(15.2)\end{array}$ & Hospitalized & 1.45 \\
\hline $\begin{array}{l}\text { Efros et al. } \\
\text { [71] }\end{array}$ & $\begin{array}{l}\text { Feb, } \\
2021\end{array}$ & Israel & Retrospective & 559 & $\begin{array}{l}\text { Myocardial injury } \\
\text { is associated with } \\
\text { mortality and death } \\
\text { within } 60 \text { days of } \\
\text { hospitalization }\end{array}$ & Mean age was 59.38 & Hospitalized & 2.1 \\
\hline $\begin{array}{l}\text { Medranda } \\
{[70]}\end{array}$ & $\begin{array}{l}\text { April, } \\
2021\end{array}$ & USA & Prospective & 2357 & $\begin{array}{l}\text { Myocardial injury } \\
\text { (troponin } \geq 1 \mathrm{ng} / \mathrm{mL} \text { ) }\end{array}$ & NR & Hospitalized & NR \\
\hline $\begin{array}{l}\text { Michielli } \\
{[78]}\end{array}$ & $\begin{array}{l}\text { April, } \\
2021\end{array}$ & USA & Retrospective & 367 & $\begin{array}{l}\text { Myocardial injury } \\
\text { was identified in } 46 \%\end{array}$ & Mean age 61 (17) & Hospitalized & 1.5 \\
\hline $\begin{array}{l}\text { Rhalete } \\
\text { [78] }\end{array}$ & $\begin{array}{l}\text { May, } \\
2021\end{array}$ & Morocco & & 610 & Myocardial injury & 65 years $(27-90)$ & ICU admitted & 2.3 \\
\hline $\begin{array}{l}\text { Ali } \\
{[66]}\end{array}$ & $\begin{array}{c}\text { March, } \\
2021\end{array}$ & Pakistan & Retrospective & 466 & Myocardial injury & $55.01 \pm 13.49$ years & Hospitalized & 2.3 \\
\hline $\begin{array}{l}\text { Tanboga } \\
{[65]}\end{array}$ & $\begin{array}{l}\text { May, } \\
2021\end{array}$ & Turkey & Retrospective & 14855 & $\begin{array}{l}\text { cTn measurements } \\
\text { within } 24 \mathrm{~h} \text { of } \\
\text { admission }\end{array}$ & $\begin{array}{l}\text { Median age } 49 \text { years } \\
(36-62)\end{array}$ & Hospitalized & 1.2 \\
\hline $\begin{array}{l}\mathrm{Wu} \\
{[55]}\end{array}$ & $\begin{array}{l}\text { May, } \\
2021\end{array}$ & China & Prospective & 40 & $\begin{array}{l}\text { Persistence of } \\
\text { myocardial injury } \\
\text { after discharge }\end{array}$ & NR & Hospitalized & NR \\
\hline $\begin{array}{l}\text { Karahan } \\
\text { [64] }\end{array}$ & $\begin{array}{l}\text { March, } \\
2021\end{array}$ & Turkey & Retrospective & 324 & $\begin{array}{l}\text { Myocardial injury } \\
\text { outcome }\end{array}$ & $\begin{array}{l}\text { Mean age } 58.4 \pm 15.2 \\
\text { years) }\end{array}$ & Hospitalized & 1.1 \\
\hline
\end{tabular}


Continue $\gg>$

\begin{tabular}{|c|c|c|c|c|c|c|c|c|}
\hline Author(s) & Year & $\begin{array}{l}\text { Country } \\
\text { of study }\end{array}$ & Study design & $\begin{array}{l}\text { Sample } \\
\text { size }\end{array}$ & Type of cardiac outcome & $\begin{array}{c}\text { Age of participants } \\
\text { (Years) }\end{array}$ & Disease severity & $\begin{array}{l}\text { Male: } \\
\text { Female } \\
\text { ratio }\end{array}$ \\
\hline $\begin{array}{l}\text { Krishna } \\
{[63]}\end{array}$ & $\begin{array}{c}\text { April, } \\
2021\end{array}$ & USA & Retrospective & 179 & $\begin{array}{l}\text { Cardiac abnormalities } \\
\text { in COVID-19 }\end{array}$ & $59.8 \pm 16.9$ years & Hospitalized & 1.3 \\
\hline $\begin{array}{l}\text { Cheng } \\
\text { [62] }\end{array}$ & $\begin{array}{l}\text { Feb, } \\
2021\end{array}$ & China & Retrospective & 1157 & Myocardial injury & $\begin{array}{l}\text { Overall mean was } \\
\text { not provided }\end{array}$ & Hospitalized & 1 \\
\hline $\begin{array}{l}\text { Guadiana- } \\
\text { Romualdo } \\
\text { [61] }\end{array}$ & $\begin{array}{l}\text { June, } \\
2021\end{array}$ & Spain & Retrospective & 1280 & Elevated troponin & $\begin{array}{l}\text { Median age (IQR): } \\
67 \text { years }\end{array}$ & Hospitalized & 1.4 \\
\hline $\begin{array}{l}\text { Chin } \\
{[60]}\end{array}$ & $\begin{array}{l}\text { Feb, } \\
2021\end{array}$ & China & Prospective & 181 & Cardiac injury & $\begin{array}{l}\text { Median age } 55 \\
\text { (IQR, 46-65) }\end{array}$ & Hospitalized & 1.3 \\
\hline $\begin{array}{l}\mathrm{He} \\
{[59]}\end{array}$ & $\begin{array}{l}\text { Feb, } \\
2022\end{array}$ & China & Retrospective & 304 & Cardiac injury & Median age, 65 years & Hospitalized & 1.1 \\
\hline $\begin{array}{l}\text { Demir } \\
\text { [58] }\end{array}$ & $\begin{array}{l}\text { May, } \\
2021\end{array}$ & UK & Retrospective & 176 & $\begin{array}{l}\text { High-Sensitivity } \\
\text { Cardiac Troponin-T } \\
\text { Concentration }\end{array}$ & $55.1 \pm 13.9$ years & Hospitalized & 2.3 \\
\hline $\begin{array}{l}\mathrm{He} \\
{[57]}\end{array}$ & $\begin{array}{l}\text { Feb, } \\
2021\end{array}$ & China & Retrospective & 173 & All with cardiac injury & $\begin{array}{l}\text { Median age } 73 \text { years } \\
\text { (IQR 64.0-80.5) }\end{array}$ & Hospitalized & 2 \\
\hline $\begin{array}{l}\text { Michilli } \\
{[54]}\end{array}$ & $\begin{array}{c}\text { April, } \\
2021\end{array}$ & USA & Retrospective & 426 & $\begin{array}{l}\text { All admitted COVID-19 } \\
\text { patients }\end{array}$ & $64.1(53.6-77.9)$ & Hospitalized & 1 \\
\hline $\begin{array}{l}\text { Briscoe } \\
{[53]}\end{array}$ & $\begin{array}{l}\text { March, } \\
2021\end{array}$ & UK & Retrospective & 346 & All cardiac patients & $\begin{array}{l}\text { Median age [IQR]; } \\
65 \text { [59-74] }\end{array}$ & Hospitalized & 2 \\
\hline $\begin{array}{l}\text { Cordeanu } \\
\text { [52] }\end{array}$ & $\begin{array}{l}\text { Dec, } \\
2020\end{array}$ & France & Retrospective & 772 & Admitted patients & $\begin{array}{l}\text { Median age was } \\
66(55-74)\end{array}$ & Hospitalized & 2 \\
\hline $\begin{array}{l}\text { Al Abbasi } \\
\text { [51] }\end{array}$ & $\begin{array}{l}\text { Dec, } \\
2020\end{array}$ & USA & Retrospective & 257 & Admitted patients & $\begin{array}{l}\text { mean age } \\
\text { of } 63 \pm 17 \text { years }\end{array}$ & Hospitalized & 2 \\
\hline $\begin{array}{l}\mathrm{Fu} \\
{[50]}\end{array}$ & $\begin{array}{l}\text { Oct, } \\
2020\end{array}$ & China & Retrospective & 355 & Admitted patients & NR & Hospitalized & 1 \\
\hline $\begin{array}{l}\text { Siddiqi } \\
\text { [50] }\end{array}$ & $\begin{array}{c}\text { April, } \\
2021\end{array}$ & USA & Prospective & 70 & Admitted patients & $\begin{array}{l}\text { median age } \\
58 \pm 17 \text { years }\end{array}$ & Hospitalized & 1.5 \\
\hline $\begin{array}{l}\text { Smilowitz } \\
{[48]}\end{array}$ & $\begin{array}{l}\text { Dec, } \\
2020\end{array}$ & USA & Retrospective & 2163 & Admitted patients & NR & Hospitalized & NR \\
\hline $\begin{array}{l}\text { Bardají } \\
\text { [47] }\end{array}$ & $\begin{array}{l}\text { Jan, } \\
2021\end{array}$ & Spain & Retrospective & 433 & Admitted patients & $67.5[52.5-77.5]$ & Hospitalized & 1.5 \\
\hline $\begin{array}{l}\text { Giustino } \\
{[46]}\end{array}$ & $\begin{array}{l}\text { Nov, } \\
2020\end{array}$ & $\begin{array}{c}\text { USA } \\
\text { and Italy }\end{array}$ & Retrospective & 305 & Admitted patients & $63(53-73)$ & Hospitalized & 2 \\
\hline $\begin{array}{l}\text { Majure } \\
{[44]}\end{array}$ & $\begin{array}{l}\text { Jan, } \\
2021\end{array}$ & USA & Retrospective & 11159 & Admitted patients & $\begin{array}{l}\text { (median (IQR)) } \\
66(56-77)\end{array}$ & Hospitalized & 1.5 \\
\hline $\begin{array}{l}\text { Al-Wahaibi } \\
\text { [43] }\end{array}$ & $\begin{array}{l}\text { Oct, } \\
2020\end{array}$ & Oman & Retrospective & 143 & Admitted patients & $49.36 \pm 15.32$ & Hospitalized & 2.5 \\
\hline $\begin{array}{l}\text { Schiavone } \\
\text { [42] }\end{array}$ & $\begin{array}{l}\text { Oct, } \\
2020\end{array}$ & italy & Prospective & 674 & Admitted patients & $60.8 \pm 15.9$ & Hospitalized & 1.5 \\
\hline $\begin{array}{l}\text { Fan } \\
{[41]}\end{array}$ & $\begin{array}{l}\text { Aug, } \\
2020\end{array}$ & China & Retrospective & 218 & Admitted patients & $61.82 \pm 16.13$ & Hospitalized & 1 \\
\hline $\begin{array}{l}\text { Ghio } \\
{[40]}\end{array}$ & $\begin{array}{l}\text { Nov, } \\
2020\end{array}$ & Italy & Prospective & 405 & Admitted patients & $\begin{array}{l}\text { Median 69.8 } \\
\text { (IQR 58.6-78.3) }\end{array}$ & Hospitalized & 2.1 \\
\hline $\begin{array}{l}\text { Heberto } \\
{[37]}\end{array}$ & $\begin{array}{l}\text { Oct, } \\
2020\end{array}$ & Mexico & Prospective & 254 & Admitted patients & $\begin{array}{l}\text { Median age was } \\
53.8 \text { SD } 12.7\end{array}$ & Hospitalized & 1.5 \\
\hline
\end{tabular}


Continue $>>$

\begin{tabular}{|c|c|c|c|c|c|c|c|c|}
\hline Author(s) & Year & $\begin{array}{l}\text { Country } \\
\text { of study }\end{array}$ & Study design & $\begin{array}{l}\text { Sample } \\
\text { size }\end{array}$ & Type of cardiac outcome & $\begin{array}{c}\text { Age of participants } \\
\text { (Years) }\end{array}$ & Disease severity & $\begin{array}{l}\text { Male: } \\
\text { Female } \\
\text { ratio }\end{array}$ \\
\hline $\begin{array}{l}\text { Cao } \\
\text { [39] }\end{array}$ & $\begin{array}{l}\text { July, } \\
2020\end{array}$ & China & Retrospective & 244 & Admitted patients & $62.58(13.43)$ & Hospitalized & 1 \\
\hline $\begin{array}{l}\text { López- } \\
\text { Otero } \\
{[38]}\end{array}$ & $\begin{array}{l}\text { Jan, } \\
2021\end{array}$ & Spain & Retrospective & 245 & Admitted patients & NR & Hospitalized & 1.5 \\
\hline $\begin{array}{l}\text { Shah } \\
{[36]}\end{array}$ & $\begin{array}{l}\text { Nov, } \\
2020\end{array}$ & Georgia & NR & 309 & Admitted patients & $\begin{array}{l}59.9 \pm 14.0 \text { (without } \\
\text { cardiac injury); } \\
68.2 \pm 14.1 \text { (with } \\
\text { cardiac injury) }\end{array}$ & Hospitalized & 1 \\
\hline $\begin{array}{l}\text { Lombardi } \\
{[35]}\end{array}$ & $\begin{array}{l}\text { Nov, } \\
2020\end{array}$ & Italy & Prospective & 614 & Admitted patients & $\begin{array}{l}\text { mean age [SD], } \\
67[13]\end{array}$ & Hospitalized & 2.3 \\
\hline $\begin{array}{l}\mathrm{Xu} \\
{[77]}\end{array}$ & $\begin{array}{l}\text { Sept, } \\
2020\end{array}$ & China & Retrospective & 102 & Admitted patients & $\begin{array}{l}\text { median age, } \\
78.0 \text { years } \\
[\mathrm{IQR}, 63.5-82.5]) \\
\end{array}$ & hospitalized & 1 \\
\hline $\begin{array}{l}\text { Saleh } \\
{[76]}\end{array}$ & $\begin{array}{l}\text { Nov, } \\
2020\end{array}$ & Iran & Prospective & 386 & Admitted patients & $59.46 \pm 15.82$ & Hospitalized & 1.5 \\
\hline
\end{tabular}

tality was $72 \%$ [56]. Of 416 COVID-19 patients, $19.7 \%$ had cardiac injury and this correlated to increase in-hospital mortality. Those patients were significantly older with comorbid conditions [67]. Of 187 COVID-19 patients, 52 (27.8\%) exhibited myocardial injury as indicated by elevated troponin $\mathrm{t}(\mathrm{TnT})$ and in-hospital mortality was $37.50 \%$ for those without history of CVD but increased levels of TnT, and $69.44 \%$ for those with a history of cardiac diseases and elevated TnTs [73]. An additional study found correlation of higher concentrations for CK-MB, NT-proBNP, MYO, and ultra-TnI with disease severity and increased in-hospital mortality [74]. Very severe COVID-19 had higher CRP and higher cardiac troponin I than severe cases [75]. One study showed that 41 $(22 \%)$ of admitted patients had myocardial injury and the mortality was similar among those with myocardial injury regardless of whether they had or did not have COVID-19 infection [47]. The included studies also examined the relationship between troponin level and mortality or the prevalence of elevated cardiac enzymes among hospitalized COVID-19 patients [35, 39, 41, 44, 51, 52, $54,58,61,66]$.

\section{DISCUSSION}

Elderly population with comorbidities are found to be at an increased risk of getting COVID-19 infections and its complications [45]. However, patients at the highest risk are those with cardiac comorbidities. COVID-19 has been linked to multiple extra-respiratory symptoms, the most important of which were cardiac manifestations, which are more prevalent in patients with positive cardiovascular disease history [74].

The present systematic review examined the medical literature during the last year from January 2020 and May 2021 to explore myocardial injury occurrence during the hospitalization time of patients with severe COVID-19 infections. The review demonstrated that the prevalence of myocardial injury was associated with higher mortality and worsened prognosis of the infections, particularly in patients with positive cardiac history and only one study showed no difference in the mortality among myocardial injury with or without COVID-19 [33, 34, 45, 47, 56, 67, 73-75].

Lorente-Ros et al. supported the notion that patients with cardiac comorbidities had a relatively higher risk of myocardial injury if they got COVID-19 infections (p value=0.005) [33]. Furthermore, Lorente-Ros et al. also categorized the risk of mortality, 30 days from the beginning of infection, based on the occurrence of MI on scoring from low, intermediate, to high risk [33]. These findings were extracted from the largest sample recruited in the whole eligible studies (707 patients).

Similarly, Guo et al. demonstrated that a positive 
history of cardiovascular disease is a strong predictor for acute myocardial injury with severe COVID-19 infection [73]. Moreover, the risk of in-hospital mortality was almost doubled in patients with a prior cardiac disease history and acute MI (69.44\%) compared to those who had an adverse history of myocardial disease with myocardial injury during infection (37.5\%). Additionally, Guo et al. confirmed that MI with positive cardiac history worsens the patient's prognosis [73].

Myocardial injury was highly related to the severity of COVID-19 infection. Zhou et al. classified 34 COVID-19 patients into severe and very severe patients, showing that the very severe group suffered from significant elevation in the troponin levels ( $p$ value $<0.001$ ) [75]. This finding was also supported by Han et al., which showed a positive and significant correlation between the COVID-19 infection severity and the elevation of cardiac enzymes [74].

Turning to the characters of COVID-19 patients at higher risk of MI, Shi, et al. revealed that patients at highest risk are significantly older ( $p$ value $<0.001$ ), with more than one comorbidities ( $p$ value $<0.001$ ), and had significantly elevated levels of inflammatory markers including C-reactive proteins, WBC count, and procalcitonin, which supports the association between the COVID-19 infection severity and the MI [67]. These findings were also compliant with the findings of Wei et al. [34]. Patients with COVID-19 can also get myocardial injury in a severe form presenting with ST-segment elevation. This was described by Bangalore et al., where $33 \%$ had chest pain, and $56 \%$ of the patients had ST-segment elevation upon admission [56]. In-hospital mortality was $72 \%$, with almost one-third of it were diagnosed with myocardial infarction. It is worth mentioning that half of the Bangalore et al. cohort had coronary angiography [56]. The largest study included 14855 patients and the 30-day mortality Hazard Ration was 1.89 (1.62-2.21) [65]. Another large study of 11159 patients looked at the elevation of cardiac enzymes and showed that 919 (15\%) had mild elevation, and 902 (14\%) had significant elevation with an adjusted OR 2.06 (95\% CI, 1.68 to 2.53; $\mathrm{p}<0.001)$ and 4.51 (95\% CI, 3.66 to 5.54; $\mathrm{p}<0.001)$, respectively [44].

On the contrary, Deng et al. showed that most of the patients were admitted with normal troponin levels, while $37.5 \%$ developed an elevation in tro- ponin levels during hospitalization [45]. This elevation in troponins was comparatively enhanced in patients who died later with COVID-19, with maximal elevation in cardiac enzyme in the week before death.

Though some aspects of the included studies could limit the review, the size of the sample of the included patients in each study is moderately small compared to the number of COVID-19 infected population; additionally, all the studies were single centered studies, which reduce the external validity of the outcomes.

Finally, although some reviews evaluated cardiac manifestations and drug-induced cardiac events in COVID-19 patients, this review focuses only on the occurrence of myocardial injury during hospitalization of COVID-19 patients with severe complications, its outcomes, and its prognosis.

\section{CONCLUSIONS}

Myocardial injury seems to be associated with severe COVID-19 disease especially among hospitalized patients and that such patients should be rigorously monitored and treated. Cardiac enzymes and inflammatory markers should be evaluated upon admission and during hospitalization in patients admitted with normal troponin due to the elevation of troponin levels during hospitalization. Patients with cardiac comorbidities should be controlled during the pandemic of COVID-19 as they have shown to be at the highest risk of myocardial injury and, subsequently, death.

\section{Ethics statement}

This is a systematic review of the past literature, so it does not require ethical consideration.

\section{Conflict of interest}

This authors have no conflicts of interest to be declared with respect to this research study.

\section{Funding}

None.

\section{REFERENCES}

[1] Zhou P, Yang X Lou, et al. A pneumonia outbreak associated with a new coronavirus of probable bat origin. Nature. 2020; 579, 270-3.

[2] Gorbalenya AE, Baker SC, Baric RS, et al. The species Severe acute respiratory syndrome-related coro- 
navirus: classifying 2019-nCoV and naming it SARSCoV-2. Nat Microbiol. 2020; 5, 536-44.

[3] Lee N, Hui D, Wu A, et al. A major outbreak of severe acute respiratory syndrome in Hong Kong. N Engl J Med. 2003; 348, 1986-94.

[4] Shaw K. The 2003 SARS outbreak and its impact on infection control practices. Public Health. 2006; 120, 8-14. [5] Zaki AM, van Boheemen S, Bestebroer TM, Osterhaus ADME, Fouchier RAM. Isolation of a novel coronavirus from a man with pneumonia in Saudi Arabia. N Engl J Med. 2012; 367, 1814-20.

[6] Corman VM, Eckerle I, Bleicker T, et al. Detection of a novel human coronavirus by real-time reverse-transcription polymerase chain reaction. Euro Surveill. 2012; 17 (39), 20285.

[7] de Groot RJ, Baker SC, Baric RS, et al. Middle East Respiratory Syndrome Coronavirus (MERS-CoV): Announcement of the Coronavirus Study Group. J Virol. 2013; 87: 7790-2.

[8] Zhou F, Yu T, Du R, et al. Clinical course and risk factors for mortality of adult inpatients with COVID-19 in Wuhan, China: a retrospective cohort study. Lancet. 2020; 6736: 1-9.

[9] Guan W, Ni Z, Hu Y, et al. Clinical characteristics of coronavirus disease 2019 in China. N Engl J Med. 2020; 382, 1708-20.

[10] Al-Omari A, Rabaan AA, Salih S, Al-Tawfiq JA, Memish ZA. MERS coronavirus outbreak: Implications for emerging viral infections. Diagn Microbiol Infect Dis. 2019; 93, 265-85.

[11] Singla R, Mishra A, Joshi R, et al. Human animal interface of SARS-CoV-2 (COVID-19) transmission: a critical appraisal of scientific evidence. Vet Res Commun. 2020; 44, 119-30.

[12] World Health Organization. WHO Coronavirus (COVID-19) Dashboard. Available at https://covid19. who.int/ (accessed April 21, 2021).

[13] Gupta A, Madhavan M V, Sehgal K, et al. Extrapulmonary manifestations of COVID-19. Nat Med. 2020; 26, 1017-32.

[14] Tirupathi R, Muradova V, Shekhar R, Salim SA, Al-Tawfiq JA, Palabindala V. COVID-19 disparity among racial and ethnic minorities in the US: A cross sectional analysis. Travel Med Infect Dis. 2020; 38, 101904.

[15] Liu PP, Blet A, Smyth D, Li H. The Science Underlying COVID-19: implications for the cardiovascular system. Circulation. 2020; 142, 68-78.

[16] Ochani RK, Asad A, Yasmin F, et al. Covid-19 pandemic: From origins to outcomes. A comprehensive review of viral pathogenesis, clinical manifestations, diagnostic evaluation, and management. Infez Med. 2021; $29,2036$.

[17] Montazerin SM, Najafi H, Shojaei F, et al. Covid19-associated coagulopathy: A concise review on pathogenesis and clinical implications. Infez Med. 2021; 29: 1-9.
[18] Tirupathi R, Ramparas TR, Wadhwa G, et al. Viral dynamics in the Upper Respiratory Tract (URT) of SARS-CoV-2. Infez Med. 2020; 28, 486-99.

[19] AlBahrani S, Al-Tawfiq JA, Alshaer AR, et al. A Case Series of Severe Hospitalized COVID-19 Patients treated with tocilizumab and glucocorticoids: a report from Saudi Arabian Hospital. J Epidemiol Glob Health. 2021; 11 (2), 233-7.

[20] Rabaan AA, Al-Ahmed SH, Muhammad J, et al. Role of inflammatory cytokines in covid-19 patients: A review on molecular mechanisms, immune functions, immunopathology and immunomodulatory drugs to counter cytokine storm. Vaccines. 2021; 9 (5), 43

[21] Eljaaly K, Malibary H, Alsulami S, Albanji M, Badawi M, Al-Tawfiq JA. Description and Analysis of Cytokine Storm in Registered COVID-19 Clinical Trials: A Systematic Review. Pathogens. 2021; 10, 692.

[22] Szekely Y, Lichter Y, Taieb P, et al. Spectrum of Cardiac Manifestations in COVID-19: a systematic echocardiographic study. Circulation. 2020; 142, 342-53.

[23] Inciardi RM, Lupi L, Zaccone G, et al. Cardiac Involvement in a Patient with Coronavirus Disease 2019 (COVID-19). JAMA Cardiol. 2020; 5, 819.24.

[24] Basu-Ray I, Soos MP. Cardiac Manifestations Of Coronavirus (COVID-19). In StatPearls, [Internet]. Treasure Island (FL): StatPearls Publishing; 2021 Jan. [25] Zheng YY, Ma YT, Zhang JY, Xie X. COVID-19 and the cardiovascular system. Nat Rev Cardiol. 2020; 17, 259-60.

[26] Kochi AN, Tagliari AP, Forleo GB, Fassini GM, Tondo C. Cardiac and arrhythmic complications in patients with COVID-19. J Cardiovasc Electrophysiol. 2020; 31, 1003-8.

[27] Fried JA, Ramasubbu K, Bhatt R, et al. The Variety of Cardiovascular Presentations of COVID-19. Circulation. 2020; 141, 1930-6.

[28] Clerkin KJ, Fried JA, Raikhelkar J, et al. COVID-19 and Cardiovascular Disease. Circulation. 2020; 141, 1648-55.

[29] Malik P, Patel U, Patel NH, Somi S, Singh J. Elevated cardiac troponin i as a predictor of outcomes in covid-19 hospitalizations: A meta-analysis. Infez Med. 2020; 28, 500-6.

[30] Lang JP, Wang X, Moura FA, Siddiqi HK, Morrow DA, Bohula EA. A current review of COVID-19 for the cardiovascular specialist. Am Heart J. 2020; 226, 29.44.

[31] Liberati A, Altman DG, Tetzlaff J, et al. The PRISMA statement for reporting systematic reviews and meta-analyses of studies that evaluate health care interventions: explanation and elaboration. PLoS Med. 2009; 6 (7), e1000100.

[32] Higgins JPT, Altman DG, Gøtzsche PC, et al. The Cochrane Collaboration's tool for assessing risk of bias in randomised trials. BMJ. 2011; 343.

[33] Lorente-Ros A, Monteagudo Ruiz JM, Rincón LM, et al. Myocardial injury determination improves risk 
stratification and predicts mortality in covid-19 patients. Cardiol J. 2020; 27, 489-96.

[34] Wei JF, Huang FY, Xiong TY, et al. Acute myocardial injury is common in patients with COVID-19 and impairs their prognosis. Heart. 2020; 106, 1154-9.

[35] Lombardi CM, Carubelli V, Iorio A, et al. Association of Troponin Levels with Mortality in Italian Patients Hospitalized with Coronavirus Disease 2019: Results of a Multicenter Study. JAMA Cardiol. 2020; 5, 1274-80.

[36] Shah P, Doshi R, Chenna A, et al. Prognostic Value of Elevated Cardiac Troponin I in Hospitalized Covid-19 Patients. Am J Cardiol. 2020; 135, 150-3.

[37] Heberto AB, Carlos PCJ, Antonio CRJ, et al. Implications of myocardial injury in Mexican hospitalized patients with coronavirus disease 2019 (COVID-19). IJC Hear Vasc. 2020; 30, 100638.

[38] López-Otero D, López-Pais J, Antúnez-Muiños PJ, Cacho-Antonio C, González-Ferrero T, González-Juanatey JR. Association between myocardial injury and prognosis of COVID-19 hospitalized patients, with or without heart disease. CARDIOVID registry. Rev Española Cardiol. English Ed. 2021; 74, 105-8.

[39] Cao J, Zheng Y, Luo Z, et al. Myocardial injury and COVID-19: Serum hs-cTnI level in risk stratification and the prediction of 30-day fatality in COVID-19 patients with no prior cardiovascular disease. Theranostics. 2020; 10, 9663-73.

[40] Ghio S, Baldi E, Vicentini A, Lenti MV, et al. Cardiac involvement at presentation in patients hospitalized with COVID-19 and their outcome in a tertiary referral hospital in Northern Italy. Intern Emerg Med. 2020; 15, 1457-65.

[41] Fan Z-X, Yang J, Zhang J, et al. Analysis of influencing factors related to elevated serum troponin I level for COVID-19 patients in Yichang, China. Cardiovasc Diagn Ther. 2020; 10, 678-86.

[42] Schiavone M, Gasperetti A, Mancone M, et al. Redefining the prognostic value of high-sensitivity troponin in COVID-19 patients: the importance of concomitant coronary artery disease. J Clin Med. 2020; 9, 3263.

[43] Al-Wahaibi K, Al-Wahshi Y, Mohamed Elfadil O. Myocardial Injury Is Associated with Higher Morbidity and Mortality in Patients with 2019 Novel Coronavirus Disease (COVID-19). SN Compr Clin Med. 2020; 2, 2514-20. [44] Majure DT, Gruberg L, Saba SG, Kvasnovsky C, Hirsch JS, Jauhar R. Usefulness of elevated troponin to predict death in patients with COVID-19 and myocardial Injury. Am J Cardiol. 2021; 138, 100-6.

[45] Deng Q, Hu B, Zhang Y, Wang H, et al. Suspected myocardial injury in patients with COVID-19: Evidence from front-line clinical observation in Wuhan, China. Int J Cardiol. 2020; 311, 116-21.

[46] Giustino G, Croft LB, Stefanini GG, et al. Characterization of myocardial injury in patients with COVID-19. J Am Coll Cardiol. 2020; 76, 2043-55.
[47] Bardají A, Carrasquer A, Sánchez-Giménez R, et al. Prognostic implications of myocardial injury in patients with and without COVID-19 infection treated in a university hospital. Rev Esp Cardiol. 2021; 74, 24-32.

[48] Smilowitz NR, Jethani N, Chen J, et al. Myocardial Injury in Adults Hospitalized with COVID-19. Circulation. 2020; 142, 2393-5.

[49] Siddiqi HK, Weber B, Zhou G, et al. Increased prevalence of myocardial injury in patients with SARSCoV-2 Viremia. Am J Med. 2021; 134, 542-6.

[50] Fu L, Li X-Y, Fei J, Xiang Y, et al. Myocardial injury at early stage and its association with the risk of death in COVID-19 Patients: a hospital-based retrospective cohort study. Front Cardiovasc Med. 2020; 7: 590688.

[51] AL Abbasi B, Torres P, Ramos-Tuarez F, et al. Cardiac Troponin-I and COVID-19: A Prognostic Tool for In-Hospital Mortality. Cardiol Res. 2020; 11, 398-404.

[52] Cordeanu E-M, Duthil N, Severac F, et al. Prognostic value of troponin elevation in COVID-19 hospitalized patients. J Clin Med. 2020; 9 (12), 4078 .

[53] Briscoe M, Sykes R, Krystofiak T, Peck O, Mangion $\mathrm{K}$, Berry C. Clinical significance of coronavirus disease 2019 in hospitalized patients with myocardial injury. Clin Cardiol. 2021; 44, 332-9.

[54] De Michieli L, Babuin L, Vigolo S, et al. Using high sensitivity cardiac troponin values in patients with SARS-CoV-2 infection (COVID-19): The Padova experience. Clin Biochem. 2021; 90, 8-14.

[55] Wu X, Deng K-Q, Li C, Yang Z, et al. Cardiac Involvement in Recovered Patients From COVID-19: A Preliminary 6-Month Follow-Up Study. Front Cardiovasc Med. 2021; 8, 654405.

[56] Bangalore S, Sharma A, Slotwiner A, et al. ST-Segment Elevation in Patients with Covid-19 - A Case Series. N Engl J Med. 2020; 382, 2478-80.

[57] He Y, Zheng X, Li X, Jiang X. Key factors leading to fatal outcomes in COVID-19 patients with cardiac injury. Sci Rep. 2021; 11 (1), 4144.

[58] Demir OM, Ryan M, Cirillo C, et al. Impact and Determinants of High-Sensitivity Cardiac Troponin-T Concentration in Patients With COVID-19 Admitted to Critical Care. Am J Cardiol. 2021; 147, 129-36.

[59] He J, Zhang B, Zhou Q, et al. The Prognostic value of myocardial injury in COVID-19 patients and associated characteristics. Res Sq. 2021 Feb 19; rs.3.rs-251810.

[60] Chen L, Hu W, Guo X, et al. Association of coagulation dysfunction with cardiac injury among hospitalized patients with COVID-19. Sci Rep. 2021; 11 (1), 4432. [61] García de Guadiana-Romualdo L, Morell-García D, Rodríguez-Fraga O, et al. Cardiac troponin and COVID-19 severity: Results from BIOCOVID study. Eur J Clin Invest. 2021; 51(6): e13532.

[62] Cheng R, Liu C, Yang J, et al. Sex Differences in the Incidence and Risk Factors of Myocardial Injury in COVID-19 Patients: A Retrospective Cohort Study. Front Physiol. 2021; 12: 632123. 
[63] Krishna H, Ryu AJ, Scott CG, Mandale DR, Naqvi TZ, Pellikka PA. Cardiac abnormalities in COVID-19 and relationship to outcome. Mayo Clin Proc. 2021; 96, 932-42. [64] Karahan S, Katkat F, Ozcan S, Sahin I, Okuyan E. Impact of acute myocardial injury on prognosis in patients with COVID-19. Eur Rev Med Pharmacol Sci. 2021; $25,2425-34$.

[65] Tanboğa IH, Canpolat U, Özcan Çetin EH, et al. The prognostic role of cardiac troponin in hospitalized COVID-19 patients. Atherosclerosis. 2021; 325: 83-8.

[66] Ali J, Khan FR, Ullah R, Hassan Z, et al. Cardiac troponin i levels in hospitalized COVID-19 patients as a predictor of severity and outcome: a retrospective cohort study. Cureus. 2021; 13.

[67] Shi S, Qin M, Shen B, et al. Association of cardiac injury with mortality in hospitalized patients with COVID-19 in Wuhan, China. JAMA Cardiol. 2020; 5 (7), 805-10. [68] El Rhalete A, Rhazi I, Bensaid A, et al. Cardiovascular injuries during COVID-19 infection: A PROCESS-compliant case series from the Eastern Morocco. Ann Med Surg. 2021; 65, 102309.

[69] De Michieli L, Ola O, Knott JD, et al. High-sensitivity cardiac troponin $\mathrm{t}$ for the detection of myocardial injury and risk stratification in COVID-19. Clin Chem. 2021; hvab062.

[70] Medranda GA, Fazlalizadeh H, Case BC, et al. Implications of left ventricular function on short-term outcomes in COVID-19 patients with myocardial injury. Cardiovasc Revascularization Med. 2021; S15538389(21)00188-3.
[71] Efros O, Barda N, Meisel E, et al. Myocardial injury in hospitalized patients with COVID-19 infection - Risk factors and outcomes. PLoS One. 2021; 16 (2), e0247800.

[72] Liaqat A, Ali-Khan RS, Asad M, Rafique Z. Evaluation of myocardial injury patterns and ST changes among critical and non-critical patients with coronavirus-19 disease. Sci Rep. 2021; 11 (1), 482.

[73] Guo T, Fan Y, Chen M, et al. Cardiovascular implications of fatal outcomes of patients with Coronavirus Disease 2019 (COVID-19). JAMA Cardiol. 2020; 5, 811-8. [74] Han H, Xie L, Liu R, et al. Analysis of heart injury laboratory parameters in 273 COVID-19 patients in one hospital in Wuhan, China. J Med Virol. 2020; 92: 819-23. [75] Zhou B, She J, Wang Y, Ma X. The clinical characteristics of myocardial injury in severe and very severe patients with 2019 novel coronavirus disease. J Infect. 2020; 81, 147-78.

[76] Karbalai Saleh S, Oraii A, Soleimani A, et al. The association between cardiac injury and outcomes in hospitalized patients with COVID-19. Intern Emerg Med. 2020; 15, 1415-24.

[77] $\mathrm{Xu} \mathrm{H}, \mathrm{Hou} \mathrm{K}, \mathrm{Xu} \mathrm{R}$, et al. Clinical characteristics and risk factors of cardiac involvement in covid-19. J Am Heart Assoc. 2020; 9 (18), e01680.

[78] McDonald I, Murray SM, Reynolds CJ, Altmann DM, Boyton RJ. Comparative systematic review and meta-analysis of reactogenicity, immunogenicity and efficacy of vaccines against SARS-CoV-2. Npj Vaccines. 2021; 6, 1-14. 